This item was submitted to Loughborough's Research Repository by the author.

Items in Figshare are protected by copyright, with all rights reserved, unless otherwise indicated.

\title{
Electronic phases in twisted bilayer graphene at magic angles as a result of Van Hove singularities and interactions
}

\section{PLEASE CITE THE PUBLISHED VERSION}

https://doi.org/10.1103/PhysRevB.98.205151

\section{PUBLISHER}

(C) American Physical Society

\section{VERSION}

VoR (Version of Record)

\section{PUBLISHER STATEMENT}

This work is made available according to the conditions of the Creative Commons Attribution-NonCommercialNoDerivatives 4.0 International (CC BY-NC-ND 4.0) licence. Full details of this licence are available at: https://creativecommons.org/licenses/by-nc-nd/4.0/

\section{LICENCE}

CC BY-NC-ND 4.0

\section{REPOSITORY RECORD}

Sherkunov, Yury, and Joseph Betouras. 2019. "Electronic Phases in Twisted Bilayer Graphene at Magic Angles as a Result of Van Hove Singularities and Interactions". figshare. https://hdl.handle.net/2134/35936. 


\title{
Electronic phases in twisted bilayer graphene at magic angles as a result of Van Hove singularities and interactions
}

\author{
Yury Sherkunov and Joseph J. Betouras* \\ Department of Physics and Centre for the Science of Materials, Loughborough University, Loughborough, LE11 3TU, United Kingdom
}

(Received 25 July 2018; published 29 November 2018)

\begin{abstract}
The discovery of different phases as a result of correlations, especially in low-dimensional materials, has been always an exciting and fundamental subject of research. Recent experiments on twisted bilayer graphene have revealed reentrant unconventional superconductivity as a function of doping as well as a Mott-like insulating phase when the two layers are twisted with respect to each other at certain "magic" angles for doping, corresponding to two particles per moiré unit cell. In this paper, we propose a microscopic model that takes into account interactions and the Van Hove singularities in the density of states of twisted bilayer graphene at doping corresponding to one particle $(v=1)$ per moiré unit cell and study how superconductivity emerges. We identify the possible symmetry of the order parameter as $s^{ \pm}$, while, if the intervalley coupling is negligible, the symmetry is $s^{++}$. In addition, we find and characterize the insulating region of the system as a region with a uniform charge instability where there is coexistence of the metallic and insulating phases.
\end{abstract}

DOI: 10.1103/PhysRevB.98.205151

\section{INTRODUCTION}

Recent experiments on twisted bilayer graphene have revealed the importance of the effects of correlations and the development of unconventional superconductivity in these two-dimensional systems [1,2]. One fundamental ingredient of this physics is that by twisting the two layers in the bilayer system with respect to each other at precisely some desired angles, the layers hybridize such as to form flatbands near the Fermi level. This, in turn, leads to Lifshitz transitions where the Fermi velocity goes to zero and the density of states (DOS) gets enhanced. Indeed, Van Hove singularities were observed in twisted bilayer graphene in an earlier paper [3]. In a broader sense, the system is then susceptible to the formation of different phases as a result of the interactions. Recent examples of the role of Lifshitz transitions in correlated systems include ferromagnetic superconductors [4], pnictides [5], and cobaltates [6].

The electronic phases that have been discovered in twisted bilayer graphene are superconductivity and a Mott-like insulator behavior in the case of hole-doped bilayer graphene with filling factor clearly at $v=2$, corresponding to two particles per unit cell of the moiré pattern. At $v=1$, there is still a debate on the nature of the insulatinglike state [7]. Deeper understanding of both phases and their relation is of fundamental importance; this has been the subject of intense studies since the discovery of high-temperature superconductors [8] and is attracting a surge of interest in relation to twisted bilayer graphene [7,9-14].

In this paper, we study the effects of correlations, taking into account the singularities in the DOS, and provide an explanation of the phase diagram in the temperature-density plane for the case of single occupation of the moiré unit

*J.Betouras@lboro.ac.uk or Y.Sherkunov@lboro.ac.uk cell, $v=1$. The physics at $v=1$ should be distinct from the one associated with higher filling factors. The reason is that according to the experimental results of Refs. [1,2], the renormalized Fermi velocity in the vicinity of the magic angle is of order of $v^{*}=4 \times 10^{4} \mathrm{~m} / \mathrm{s}$, which is 25 times smaller than the one of a single-layer graphene. This puts the position of the Van Hove singularities in question at $\epsilon_{0}=0.25 \mathrm{meV}$, which corresponds to the filling factor $v=1$ per moiré unit cell [13]. In addition, there is no nesting at that filling, contrary to the structure of the singularities associated with higher filling factors.

We find that the system shows reentrant behavior of the superconductivity, for which we predict that the order parameter symmetry is $s^{ \pm}$or $s^{++}$, therefore different than the one predicted for single-layer graphene $[15,16]$ and we provide the reason for that. We also find a phase of uniform charge instability (UCI), with coexistence of insulating and metallic regions.

The structure of the paper is that in the next section we provide a discussion on the effective Hamiltonian; in Sec. III there is a discussion on the polarization operators and the structure of RG equations; in Sec. IV the results of RG analysis are presented and, finally, in Sec. V, we discuss the results in the context of the experimental work.

\section{EFFECTIVE HAMILTONIAN}

A twisted bilayer graphene has a moiré superlattice pattern, which is reciprocal to a hexagonal mini Brillouin zone, with side, $\Delta K=K \theta$, equal to the difference between two $K$-vectors of the twisted layers as shown in Fig. 1(a). The electronic spectrum of twisted bilayer graphene has been thoroughly studied [17-19]. A continuous low-energy theory developed in Ref. [20], with interlayer tunneling only between the Dirac points of the mini Brillouin zone parameterized by vectors $\mathbf{q}_{1,2,3}$ generating a $k$-space honeycomb lattice 
(a)

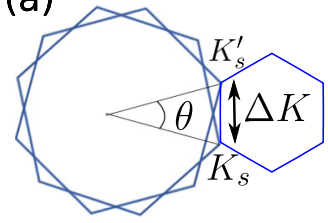

(b)

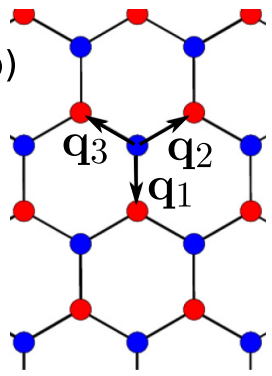

FIG. 1. Momentum-space geometry of a bilayer graphene twisted by angle $\theta$. (a) The Brillouin zones of the two graphene layers are shown on the left and the mini Brillouin zones of the twisted bilayer graphene constructed from the difference, $\Delta \mathbf{K}=\mathbf{K}_{s}-\mathbf{K}_{s}^{\prime}$, between the two vectors of the Dirac points, $\mathbf{K}_{s}$ and $\mathbf{K}_{s}^{\prime}$, of the two layers are shown on the right. At small angles, $\Delta K=\left|\mathbf{K}_{s}\right| \theta$. (b) The three equivalent Dirac points in the first mini Brillouin zone result in three distinct hopping processes described by vectors $\mathbf{q}_{1}=$ $\Delta K(0,-1), \mathbf{q}_{2}=\Delta K(\sqrt{3} / 2,1 / 2)$, and $\mathbf{q}_{3}=\Delta K(-\sqrt{3} / 2,1 / 2)$, generating a honeycomb lattice in the $k$-space.

corresponding to repeated hopping, as shown in Fig. 1(b). We adopt this theory to study the low-energy spectrum and derive the effective Hamiltonian as a starting point.

In the simplest limit in which the momentum-space lattice is truncated to the first honeycomb shell, the system can be

described by the following Hamiltonian [20]:

$$
H=\left[\begin{array}{cccc}
h_{k} & T_{1} & T_{2} & T_{3} \\
T_{1}^{\dagger} & h_{k_{1}} & 0 & 0 \\
T_{2}^{\dagger} & 0 & h_{k_{2}} & 0 \\
T_{3}^{\dagger} & 0 & 0 & h_{k_{3}}
\end{array}\right],
$$

where $h_{k_{i}}=-v \boldsymbol{\sigma}^{*} \cdot \mathbf{k}_{i}$ is the Dirac Hamiltonian in the vicinity of one of the four Dirac points of the first honeycomb shell connected by the vectors $\mathbf{q}_{i}$, as shown in Fig. 1(b), $\mathbf{k}_{i}=\mathbf{k}+\mathbf{q}_{i}, v$ is the bare Fermi velocity, and $\sigma$ is a vector of Pauli matrices. The tunneling matrix elements are given by

$$
T_{i}=w\left[\begin{array}{cc}
e^{-i \phi_{i}} & 1 \\
e^{i \phi_{i}} & e^{-i \phi_{i}}
\end{array}\right],
$$

where $w$ is the hopping energy $\phi_{1}=0, \phi_{2}=2 \pi / 3$, and $\phi_{3}=-2 \pi / 3$.

In first-order perturbation theory in $k$, the effective lowenergy Hamiltonian is written as [20]

$$
H_{1}=-v^{*} \boldsymbol{\sigma}^{*} \cdot \mathbf{k},
$$

where $v^{*}$ is the renormalized Fermi velocity given by $v^{*}=$ $v \frac{1-3 \alpha^{2}}{1+6 \alpha^{2}}$, where $\alpha=w /(v \Delta K)$. For $\alpha=\alpha_{0}=1 / \sqrt{3}, v^{*}$ vanishes, leading to the flattening of the low-energy bands. This happens at one of the "magic" twist angles. Then, using second-order perturbation theory in $k$, the next order of the effective Hamiltonian reads

$$
H_{2}=\frac{3 \alpha^{2} v}{\left(1+6 \alpha^{2}\right) \Delta K k}\left[\begin{array}{cc}
k_{x}\left(k_{x}^{2}-3 k_{y}^{2}\right) & \left(k_{x}+i k_{y}\right)\left(3 k_{x}^{2}-k_{y}^{2}\right) k_{y} / k \\
\left(k_{x}-i k_{y}\right)\left(3 k_{x}^{2}-k_{y}^{2}\right) k_{y} / k & -k_{x}\left(k_{x}^{2}-3 k_{y}^{2}\right)
\end{array}\right] .
$$

Diagonalizing the Hamiltonian $H=H_{1}+H_{2}$, we find the eigenvalues, which coincide with the ones obtained from a phenomenological $k$-expansion of the low-energy Hamiltonian [1],

$$
\epsilon= \pm \sqrt{v^{* 2} k^{2}-\frac{v^{*} k^{3} \sin (3 \beta)}{m}+\frac{k^{4}}{4 m^{2}}}
$$

where $\beta$ is the angle between $\mathbf{k}$ and $k_{x}$; however, our approach enabled us to identify the parameter $m$ as $m=\frac{\left(1+6 \alpha^{2}\right) \Delta K}{6 \alpha^{2} v}$.

The energy spectrum Eq. (4) has three saddle points, as shown in Fig. 2(a), located at $\left\{k_{\mathrm{xsp}}, k_{\mathrm{ysp}}\right\}$ with $\left|k_{\mathrm{sp}}\right|=m\left|v^{*}\right|$. In the vicinity of the saddle points, the energy can be expanded as

$$
\begin{aligned}
\epsilon_{1} & =\frac{1}{2 m}\left(9 \delta k_{x}^{2}-\delta k_{y}^{2}\right), \\
\epsilon_{2 / 3} & =\frac{1}{4 m}\left(3 \delta k_{x}^{2} \mp 10 \sqrt{3} \delta k_{x} \delta k_{y}+13 \delta k_{y}^{2}\right),
\end{aligned}
$$

where $\delta k_{j}=k_{j}-k_{\text {jsp }}$. The values of the energies are counted as the difference from their values at the saddle points, $\epsilon_{0}=m v^{* 2} / 2$, which can be absorbed into the chemical potential. The azimuthal positions of the saddle points are determined by the valley index and the sign of $v^{*}$, so that for the valley $K_{s}$ and $v^{*}>0, \beta_{\mathrm{sp}}=\pi / 6,5 \pi / 6,3 \pi / 2$. For $K_{s}^{\prime}$ valley or $v^{*}<0$, the positions can be obtained by inversion, $\mathbf{k}_{\mathrm{sp}} \rightarrow-\mathbf{k}_{\mathrm{sp}}$. Thus, for each saddle point in valley $K_{s}$, there is a conjugate saddle point with identical energy in valley $K_{s}^{\prime}$, as shown in Fig. 2(b). The saddle points shift toward the point $k=0$, as $\left|v^{*}\right|$ decreases, where they merge at $v^{*}=0$ to form a minimum, as shown in the middle panel of Fig. 2(b), in contrast to Ref. [21], where, by controlling the gate voltage, the three saddle points merge to form a monkey saddle point, leading to a power-law singularity in the DOS.

\section{POLARIZATION OPERATORS AND RG ANALYSIS}

The presence of the saddle points leads to a logarithmically divergent Van Hove singularity in the DOS per spin, per saddle point,

$$
v(\epsilon)=v_{0} \ln \left|\frac{\Lambda}{\epsilon}\right|
$$

where $v_{0}=\frac{8 m}{9 \sqrt{3} \Delta K^{2}}$, and $\Lambda$ is the usual ultraviolet cutoff. Note that at $v^{*}=0$, the saddle points merge into a minimum and the DOS becomes constant.

Due to the logarithmic divergence of the DOS and the polarization operators (shown below), the renormalization group (RG) theory is the major tool at work. We follow the standard procedure developed in Refs. [22,23] that has been also used in monolayer graphene doped up to the M-points [16]. In this procedure, the fermions that are taken into account are those 


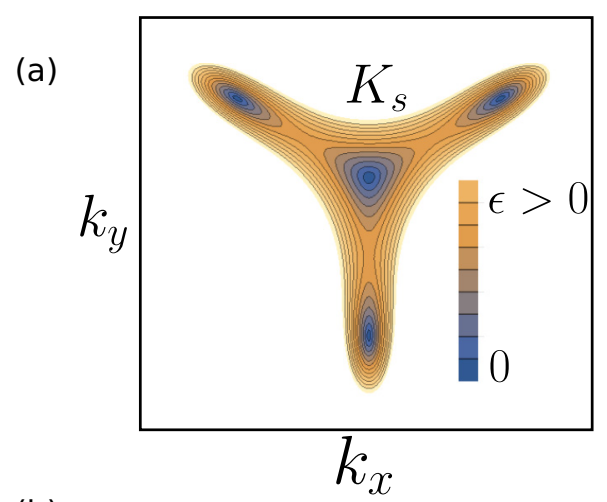

(b)

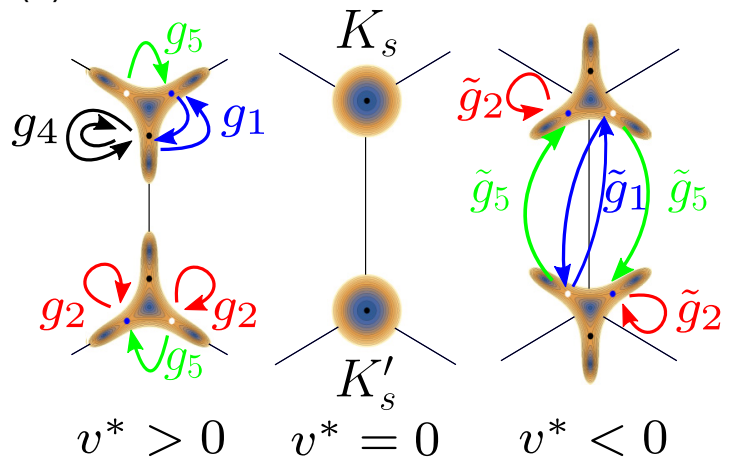

FIG. 2. (a) Low-energy electronic dispersion in the vicinity of a $K$ point of TBG for $\epsilon>0$ and $v^{*}>0$. Contours show the Fermisurface family, with Lifshitz transition occurring when the chemical potential crosses the saddle points. (b) Pictorial representation of the scattering processes shown on the plot of energy dispersion for two nonequivalent Dirac points of TBG for $v^{*}>0$ (left), $v^{*}=0$ (middle), and $v^{*}<0$ (right). Dots of the same color indicate the saddle points with identical energy dispersion (conjugate points).

that live in patches around each of the six saddle points with logarithmically divergent DOS.

It is worth emphasizing again that the renormalized Fermi velocity in the vicinity of the magic angle is of order of $v^{*}=4 \times 10^{4} \mathrm{~m} / \mathrm{s}[1,2]$, thus 25 times smaller than the one of single-layer graphene. As a result, the position of the Van Hove singularities is at $\epsilon_{0}=0.25 \mathrm{meV}$, corresponding to the filling factor $v=1$. We will adopt this value for our calculations. Moreover, there is no nesting in this case.

The screening of the Coulomb interaction, $U(k)=$ $2 \pi e^{2} /\left(k a^{2}\right)$, due to high-energy states can be estimated using random phase approximation [16]:

$$
U(k)=\frac{2 \pi e^{2} /\left(k a^{2}\right)}{1+2 \pi e^{2} N \Pi_{\Lambda_{0}} /\left(k a^{2}\right)},
$$

where $a$ is the carbon-carbon distance in a monolayer graphene, $N=12$ is the number of fermionic flavors, and $\Pi_{\Lambda_{0}}$ is the polarazation operator taking into account all the states between some ultraviolet cutoff, $\Lambda_{0}$, and the band-width, $W$. For large $k$, it can be estimated as [16] $\Pi_{\Lambda_{0}}(k) \approx \nu\left(\Lambda_{0}\right)=$ $v_{0} \ln \left|\Lambda / \Lambda_{0}\right|$. This allows us to rewrite Eq. (7) as

$$
v_{0} U(k)=\frac{Z(k)}{1+N Z(k)},
$$

with $Z(k)=2 \pi e^{2} v_{0} /\left(k a^{2}\right)$, where we assumed $\Lambda_{0}=\Lambda$. The two-particle scattering between patches is determined by the following characteristic momenta: $k_{1} \approx m v^{*} / 3$ for intrapatch (a)

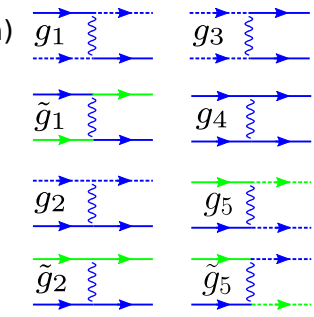

(b)

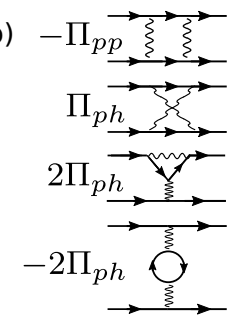

(c)

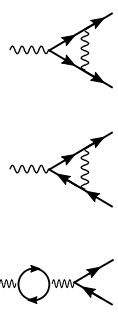

FIG. 3. (a) Feynman diagrams representing two-particle scattering processes between different patches [see Fig. 2(b)]. Solid and dashed lines represent fermions on different patches, with different colors marking two patches of different valleys with identical energies (conjugate points). Wavy lines represent interactions. Note that the Umklapp scattering, $g_{3}$, is forbidden due to momentum conservation. (b), (c) One-loop contributions to the renormalization of the interaction constants (b) and test vertices (c).

scattering, $k_{2} \approx m v^{*}$ for interpatch scattering within the same valley, and $k_{3} \approx \Delta K$ for intervalley scattering, for which we estimate $Z\left(k_{1}\right) \approx \frac{10 \sqrt{3} v}{\pi v^{*} \theta^{2}}, Z\left(k_{2}\right)=Z\left(k_{1}\right) / 3$, and $Z_{3} \approx \frac{5 \sqrt{3}}{\pi \theta^{2}}$. Here we used $e^{2} / v \approx 10 / 3$, for $a \approx 1 \AA$ and $v \approx 10^{6} \mathrm{~m} / \mathrm{s}$. For $v^{*} \ll v$ and $\theta \approx 1^{\circ}, Z\left(k_{i}\right) N \gg 1$ for $i=1,2,3$, and the Coulomb potential is completely screened, so that $\nu_{0} U\left(k_{1}\right)=$ $v_{0} U\left(k_{2}\right)=v_{0} U\left(k_{3}\right)=1 / N \approx 0.083$. This allows us to assume that the coupling is valley independent.

The two-particle scattering between patches is described by the eight distinct interactions in the low-energy theory depicted in Fig. 3(a) and visualized in Fig. 2(b). The system is described by the low-energy Lagrangian:

$$
\begin{aligned}
\mathcal{L}= & \sum_{\alpha, \sigma} \psi_{\alpha \sigma}^{\dagger}\left(\partial_{\tau}-\epsilon_{k}+\mu\right) \psi_{\alpha \sigma} \\
& -\frac{1}{2} \sum_{\alpha, \beta, \sigma, \sigma^{\prime}}\left[g_{1} \psi_{\alpha \sigma}^{\dagger} \psi_{\beta \sigma^{\prime}}^{\dagger} \psi_{\alpha \sigma^{\prime}} \psi_{\beta \sigma}+g_{2} \psi_{\beta \sigma}^{\dagger} \psi_{\alpha \sigma^{\prime}}^{\dagger} \psi_{\alpha \sigma^{\prime}} \psi_{\beta \sigma}\right. \\
& +g_{3} \psi_{\alpha \sigma}^{\dagger} \psi_{\alpha \sigma^{\prime}}^{\dagger} \psi_{\beta \sigma^{\prime}} \psi_{\beta \sigma}+g_{5} \psi_{\beta \sigma}^{\dagger} \psi_{\beta^{\prime} \sigma^{\prime}}^{\dagger} \psi_{\alpha^{\prime} \sigma^{\prime}} \psi_{\alpha \sigma} \\
& \left.+\tilde{g}_{5} \psi_{\beta \sigma}^{\dagger} \psi_{\alpha^{\prime} \sigma^{\prime}}^{\dagger} \psi_{\beta^{\prime} \sigma^{\prime}} \psi_{\alpha \sigma}\right]-\frac{1}{2} \sum_{\alpha, \sigma, \sigma^{\prime}}\left[\tilde{g}_{1} \psi_{\alpha^{\prime} \sigma}^{\dagger} \psi_{\alpha \sigma^{\prime}}^{\dagger} \psi_{\alpha^{\prime} \sigma^{\prime}} \psi_{\alpha \sigma}\right. \\
& \left.+\tilde{g}_{2} \psi_{\alpha \sigma}^{\dagger} \psi_{\alpha^{\prime} \sigma^{\prime}}^{\dagger} \psi_{\alpha^{\prime} \sigma^{\prime}} \psi_{\alpha \sigma}+g_{4} \psi_{\alpha \sigma}^{\dagger} \psi_{\alpha \sigma^{\prime}}^{\dagger} \psi_{\alpha \sigma^{\prime}} \psi_{\alpha \sigma}\right]
\end{aligned}
$$

where $\alpha$ and $\beta$ are patch indices, $\alpha^{\prime}$ labels the patch conjugate to $\alpha, \sigma=\uparrow, \downarrow$ is the spin index. The sum over $\alpha$ and $\beta$ is taken over only nonconjugate patches. Note that the Umklapp scattering, $g_{3}$, is forbidden because it does not conserve momentum modulo a reciprocal lattice vector [21].

The building blocks of the RG analysis are the polarization operators in the particle-particle and particle-hole channels, shown in the two top diagrams of Fig. 3(b), respectively, at zero momentum transfer and at momentum transfer $\mathbf{Q}_{\alpha \beta}$ between two patches $\alpha$ and $\beta$. They can be calculated as

$$
\begin{array}{r}
\Pi_{p p}(\mathbf{q})=T \int_{p} G\left(i \omega_{n}, \mathbf{p}+\mathbf{q}\right) G\left(-i \omega_{n},-\mathbf{p}\right), \\
\Pi_{p h}(\mathbf{q})=-T \int_{p} G\left(i \omega_{n}, \mathbf{p}+\mathbf{q}\right) G\left(i \omega_{n}, \mathbf{p}\right),
\end{array}
$$


where $\quad \int_{p} \ldots=\sum_{n} \int d^{2} p, \quad \omega_{n}=\pi(2 n+1) T, \quad$ and $G\left(\mathbf{p}, i \omega_{n}\right)=\left[i \omega_{n}-\epsilon(\mathbf{p})+\mu\right]^{-1}$ is the fermionic Matsubara Green's function. For energies Eqs. (5), the polarization operators can be evaluated as

$$
\begin{gathered}
\Pi_{p h}\left(\mathbf{Q}_{\alpha, \alpha^{\prime}}\right)=\Pi_{p h}(0)=v_{0} \ln \left(\frac{\Lambda}{\max \{T,|\mu|\}}\right) \\
\Pi_{p h}\left(\mathbf{Q}_{\alpha, \beta}\right)=A v_{0} \ln \left(\frac{\Lambda}{\max \{T,|\mu|\}}\right) \\
\Pi_{p p}\left(\mathbf{Q}_{\alpha, \alpha^{\prime}}\right)=\Pi_{p p}(0)=\frac{v_{0}}{2} \ln \left(\frac{\Lambda}{\max \{T,|\mu|\}}\right) \ln \left(\frac{\Lambda}{T}\right), \\
\Pi_{p p}\left(\mathbf{Q}_{\alpha, \beta}\right)=B v_{0} \ln \left(\frac{\Lambda}{\max \{T,|\mu|\}}\right)
\end{gathered}
$$

where $\quad A=\frac{\sqrt{3}}{5} \ln \left(\frac{37+20 \sqrt{3}}{13}\right) \approx 0.59 \quad$ and $\quad B=$ $\frac{6}{\sqrt{3} 9}\left[\pi-\arctan \left(2 \sqrt{\frac{3}{13}}\right)\right] \approx 2.28$. Note that the polarization operators at the momentum transfer connecting two conjugate saddle points are equal to the ones at zero momentum transfer due to energy degeneracy. Similar to the case of monolayer graphene doped to the saddle (M-) points, $\Pi_{p p}(0) \propto \ln [\Lambda / T]^{2}$ at $\mu \ll T[16,24]$; however, in the present case we find that $\Pi_{p h}\left(\mathbf{Q}_{\alpha, \beta}\right)$ is linear in $\ln (\Lambda / T)$ contrary to the monolayer graphene, where the dependence can be quadratic. The difference is because, in the case of twisted bilayer graphene at filling factor 1, there is no nesting in the Fermi surfaces, again in contrast with monolayer graphene. The relevant Feynman diagrams are shown in Figs. 3(a) and 3(b). We then obtain the following RG equations in one-loop approximation:

$$
\begin{gathered}
\frac{d g_{1}}{d y}=2 d_{0} g_{1}\left(g_{1}+\tilde{g}_{1}+g_{4}\right)+2 d_{1}\left[g_{1}\left(g_{2}-g_{1}\right)\right. \\
\left.+g_{5}\left(\tilde{g}_{5}-g_{5}\right)\right]-2 d_{3} g_{1} g_{2} \\
\frac{d \tilde{g}_{1}}{d y}=d_{0}\left[4 g_{1}^{2}+2 \tilde{g}_{1}\left(\tilde{g}_{2}+g_{4}-\tilde{g}_{1}\right)\right] \\
\quad-2 d_{4}(y)\left(\tilde{g}_{1} \tilde{g}_{2}+g_{5} \tilde{g}_{5}\right) \\
\frac{d g_{2}}{d y}=2 d_{0}\left[g_{2}\left(\tilde{g}_{1}-2 \tilde{g}_{2}-2 g_{2}-g_{4}\right)+g_{1}\left(\tilde{g}_{2}+g_{4}\right)+2 g_{1} g_{2}\right] \\
+2 d_{1}\left(g_{2}^{2}+\tilde{g}_{5}^{2}\right)-2 d_{3}\left(g_{1}^{2}+g_{2}^{2}\right) \\
\frac{d \tilde{g}_{2}}{d y}=d_{0}\left[\tilde{g}_{2}\left(\tilde{g}_{2}-2 g_{4}\right)+8 g_{2}\left(g_{1}-g_{2}\right)+2 \tilde{g}_{1} g_{4}\right] \\
-d_{4}(y)\left(\tilde{g}_{1}^{2}+\tilde{g}_{2}^{2}+2 g_{5}^{2}+2 \tilde{g}_{5}^{2}\right) \\
\frac{d g_{4}}{d y}= \\
d_{0}\left[g_{4}^{2}-8 g_{2}^{2}-2 \tilde{g}_{2}^{2}+4 g_{1}\left(g_{1}+2 g_{2}\right)\right. \\
\left.\quad+\tilde{g}_{1}\left(\tilde{g}_{1}+2 \tilde{g}_{2}\right)\right]-d_{4}(y) g_{4}^{2}
\end{gathered}
$$
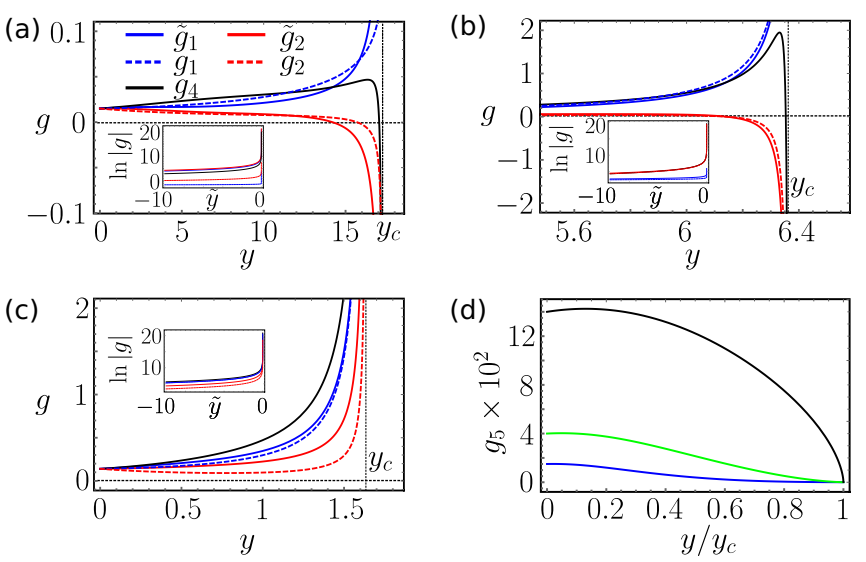

FIG. 4. (a)-(c) Flow of the coupling constants with renormalization group scale $y$ starting from repulsive interaction $g_{i}(0)=g_{0}>0$ for (a) $g_{0}=0.015$; (b) $g_{0}=0.04$, and (c) $g_{0}=0.14$. The chemical potential $\mu=0$ corresponds to the Van Hove filling. Insets: $\ln \left|g_{i}\right|$ as a function of $\tilde{y}=10^{4}\left(y-y_{c}\right)$ in the vicinity of $y_{c}$, demonstrating $\left|g_{1}\right|,\left|g_{2}\right| \ll\left|g_{4}\right|,\left|\tilde{g}_{1}\right|,\left|\tilde{g}_{2}\right|$ in case (a) and $\left|g_{1}\right|,\left|\tilde{g}_{1}\right| \ll\left|g_{2}\right|,\left|\tilde{g}_{2}\right|,\left|g_{4}\right|$ in case (b). (d) $g_{5}=\tilde{g}_{5}$ as a function of $y / y_{c}$ for $g_{0}=0.015$ (blue), $g_{0}=0.04$ (green), and $g_{0}=0.14$ (black).

$$
\begin{aligned}
\frac{d g_{5}}{d y}= & 2 d_{1}\left[2 g_{2} g_{5}+g_{1}\left(\tilde{g}_{5}-2 g_{5}\right)\right] \\
& -d_{4}(y)\left(2 \tilde{g}_{1} \tilde{g}_{5}+2 \tilde{g}_{2} g_{5}+g_{5}^{2}+\tilde{g}_{5}^{2}\right), \\
\frac{d \tilde{g}_{5}}{d y}= & 2 d_{1}\left[2 g_{2} \tilde{g}_{5}+g_{1}\left(g_{5}-2 \tilde{g}_{5}\right)\right] \\
& -d_{4}(y)\left(2 \tilde{g}_{1} g_{5}+2 \tilde{g}_{2} \tilde{g}_{5}+g_{5}^{2}+\tilde{g}_{5}^{2}\right),
\end{aligned}
$$

where $y=\Pi_{p h}(0) / \nu_{0}$ and $g_{i} \rightarrow g_{i} \nu_{0}$ are dimensionless. We also define $d_{0}=1, d_{1}=\frac{d \Pi_{p h}\left(\mathbf{Q}_{\alpha \beta}\right)}{d \Pi_{p h}(0)}=A, d_{3}=\frac{d \Pi_{p p}\left(\mathbf{Q}_{\alpha \beta}\right)}{d \Pi_{p h}(0)}=B$, and $d_{4}(y)=\frac{d \Pi_{p p}(0)}{d \Pi_{p h}(0)}=\ln (\Lambda / T) / 2 \equiv x / 2$. Given that, for $T \gg|\mu|, x=y$, and, for $T \ll|\mu|, x \gg y=\ln (\Lambda /|\mu|)$, we interpolate $d_{4}(y)$ as

$$
d_{4}(y)=\frac{y z}{2(z-y)},
$$

where $z=\ln (\Lambda /|\mu|)$.

An alternative way to get the RG equations, with a set of different assumptions, is presented in Appendix A. This procedure retains the structure of the RG equations with a different definition of the coefficients and leads to the same physical results. For completeness, all the results are included in Appendix A.

\section{RESULTS OF THE RG ANALYSIS}

In Fig. 4, we show the numerical solutions of Eqs. (16)(22) for $g_{i}(0)=g_{0}>0$ and $\mu=0$. We found that $g_{5}$ and $\tilde{g}_{5}$ are irrelevant [see Fig. 4(d)]. The remaining couplings diverge at a scale $y_{c} \propto g_{0}^{-1 / 2}$. For $g_{0}<g_{0}^{(1)} \approx 0.024$, the main contribution comes from the scattering between conjugate points described by $\tilde{g}_{1}$ and $\tilde{g}_{2}$, as well as intrapatch interaction $g_{4}$, with $\tilde{g}_{1}$ flowing to repulsion and $\tilde{g}_{2}$ and $g_{4}$ flowing to attraction, as shown in Fig. 4(a). For $g_{0}^{(1)}<g_{0}<g_{0}^{(2)} \approx$ 


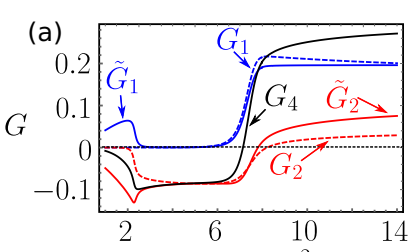

(b)
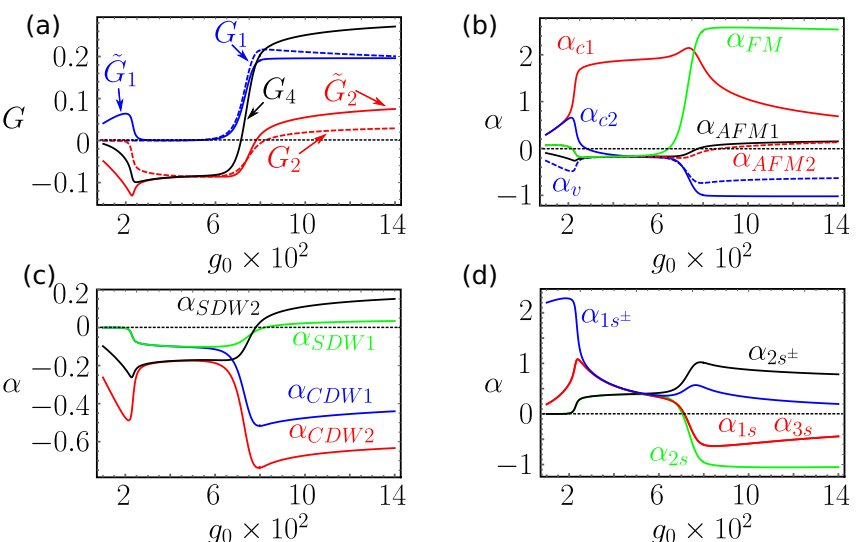

(d)

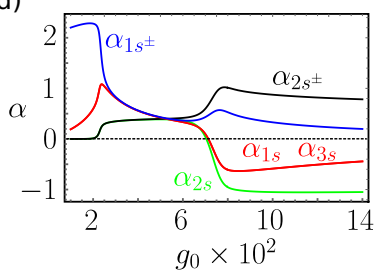

FIG. 5. Parameters of susceptibilities at the Van Hove filling for $g_{i}(0)=g_{0}>0$. (a) $G_{i}$ as functions of $g_{0}$. (b)-(d) $\alpha_{i}$ as functions of $g_{0}$ for (b) uniform densities, (c) spin and charge density waves, and (d) superconductivity.

0.075 , the main contribution arises from $\tilde{g}_{2}, g_{2}$, and $g_{4}$, which flow to attraction, as shown in Fig. 4(b). At $g_{0}>g_{0}^{(2)}$, all contributions are of the same order; however, the parameters $g_{i}$ flow to repulsion [see Fig. 4(c)]. To study the couplings for the whole range of $y$, we note that close to $y_{c}$, the relevant coupling can be cast as $g_{i}=G_{i} /\left(y_{c}-y\right)$. Substituting it into Eqs. (16)-(20), we find that $G_{i}$ satisfies simultaneous polynomial equations, which can be solved numerically to confirm our observations, as we show in Fig. 5(a).

The nature of instabilities can be identified with the help of the relevant susceptibilities $[16,21,22]$ as the most divergent susceptibility corresponds to the leading instability. Presenting the susceptibilities, $\chi_{i}$, close to $y_{c}$, as $\chi_{i}=\left(y_{c}-y\right)^{-\alpha_{i}}$, the leading instability can be found as the one with maximal positive $\alpha_{i}$.

We then introduce infinitesimal test vertices and study their renormalization, described by three-leg diagrams in Fig. 3(c). The test vertex for the instabilities due to uniform densities, $\delta \mathcal{L}=\sum_{\sigma \alpha} n_{\sigma, \alpha} \psi_{\sigma \alpha}^{\dagger} \psi_{\sigma \alpha}$, can be renormalized in one-loop approximation according to

$$
\begin{aligned}
\frac{d n_{\sigma \alpha}}{d y}= & d_{0}\left\{-g_{4} n_{\bar{\sigma} \alpha}+\left(\tilde{g}_{1}-\tilde{g}_{2}\right) n_{\sigma \alpha^{\prime}}-\tilde{g}_{2} n_{\bar{\sigma} \alpha^{\prime}}\right. \\
& \left.+\sum_{\beta \neq \alpha, \alpha^{\prime}}\left[\left(g_{1}-g_{2}\right) n_{\sigma \beta}-g_{2} n_{\bar{\sigma} \beta}\right]\right\},
\end{aligned}
$$

where $\bar{\sigma}=-\sigma$, and primes again mark conjugate saddle points. The right-hand side of Eq. (24) can be cast in a $12 \times 12$ matrix in the basis $\left\{n_{\uparrow 1}, n_{\downarrow 1}, n_{\uparrow 1^{\prime}}, n_{\downarrow 1^{\prime}} \ldots\right\}$, whose eigenvalues, $\gamma_{i}$, are related to the susceptibilities as $\alpha_{i}=2 \gamma_{i}$. We find six distinct eigenvalues corresponding to charge, $\alpha_{c 1}$ and $\alpha_{c 2}$, valley, $\alpha_{v}$, antiferromagnetic, $\alpha_{\mathrm{AFM} 1}$ and $\alpha_{\mathrm{AFM} 2}$, and ferromagnetic, $\alpha_{\mathrm{FM}}$, instabilities:

$$
\begin{gathered}
\alpha_{c 1}=2 d_{0}\left(\tilde{G}_{1}-2 \tilde{G}_{2}-G_{4}+4 G_{1}-8 G_{2}\right), \\
\alpha_{c 2}=2 d_{0}\left(\tilde{G}_{1}-2 \tilde{G}_{2}-G_{4}-2 G_{1}+4 G_{2}\right), \\
\alpha_{v}=2 d_{0}\left(-\tilde{G}_{1}+2 \tilde{G}_{2}-G_{4}\right), \\
\alpha_{\mathrm{AFM} 1}=2 d_{0}\left(-\tilde{G}_{1}+G_{4}\right),
\end{gathered}
$$

$$
\begin{gathered}
\alpha_{\mathrm{AFM} 2}=2 d_{0}\left(\tilde{G}_{1}+G_{4}-2 G_{1}\right), \\
\alpha_{\mathrm{FM}}=2 d_{0}\left(\tilde{G}_{1}+G_{4}+4 G_{1}\right) .
\end{gathered}
$$

Next we turn to spin-density wave (SDW) and charge-density wave $(\mathrm{CDW})$ instabilities, for which the test vertex, $\delta \mathcal{L}=$ $\sum_{\sigma \mathbf{Q}} n_{\sigma \mathbf{Q}} \psi_{\sigma \beta}^{\dagger} \psi_{\sigma \alpha}+$ H.c., where we use $\mathbf{Q} \equiv \mathbf{Q}_{\alpha \beta}$. The renormalization of $n_{\sigma \mathbf{Q}}$ can be obtained from the one-loop equations for nonconjugate patches,

$$
\frac{d n_{\sigma \mathbf{Q}}}{d y}=d_{1}\left[\left(g_{2}-g_{1}\right) n_{\sigma \mathbf{Q}}-g_{1} n_{\sigma \mathbf{Q}}\right]
$$

while for the conjugate ones,

$$
\frac{d n_{\sigma \mathbf{Q}^{\prime}}}{d y}=d_{0}\left[\left(\tilde{g}_{2}-\tilde{g}_{1}\right) n_{\sigma \mathbf{Q}^{\prime}}-\tilde{g}_{1} n_{\sigma \mathbf{Q}^{\prime}}\right],
$$

where in our notation $\mathbf{Q}^{\prime} \equiv \mathbf{Q}_{\alpha \alpha^{\prime}}$. These equations yield CDW and SDW instabilities as

$$
\begin{gathered}
\alpha_{\mathrm{CDW} 1}=2 d_{1}\left(-2 G_{1}+G_{2}\right), \\
\alpha_{\mathrm{CDW} 2}=2 d_{0}\left(-2 \tilde{G}_{1}+\tilde{G}_{2}\right), \\
\alpha_{\mathrm{SDW} 1}=2 d_{1} G_{2}, \\
\alpha_{\mathrm{SDW} 2}=2 d_{0} \tilde{G}_{2},
\end{gathered}
$$

where index 1 (2) corresponds to the density waves developed on nonconjugate (conjugate) patches.

To study superconductivity we introduce intra- and interpatch vertices, $\delta \mathcal{L}_{\text {intra }}=\sum_{\alpha} \Delta_{\alpha} \psi_{\uparrow \alpha} \psi_{\downarrow \alpha}+$ H.c., and $\delta \mathcal{L}_{\text {inter }}=$ $\sum_{\mathbf{Q}}\left(\Delta_{\mathbf{Q}}^{(1)} \psi_{\uparrow \alpha} \psi_{\downarrow \beta}+\Delta_{\mathbf{Q}}^{(2)} \psi_{\uparrow \beta} \psi_{\downarrow \alpha}\right)+$ H.c., which renormalize according to the following equation:

$$
\frac{d \Delta_{\alpha}}{d y}=-d_{4} G_{4} \Delta_{\alpha}
$$

and $(j \neq i)$ :

$$
\begin{aligned}
& \frac{d \Delta_{\mathbf{Q}}^{(i)}}{d y}=-d_{3}\left(G_{2} \Delta_{\mathbf{Q}}^{(i)}+G_{1} \Delta_{\mathbf{Q}}^{(j)}\right), \\
& \frac{d \Delta_{\mathbf{Q}^{\prime}}^{(i)}}{d y}=-d_{4}\left(\tilde{G}_{2} \Delta_{\mathbf{Q}^{\prime}}^{(i)}+\tilde{G}_{1} \Delta_{\mathbf{Q}^{\prime}}^{(j)}\right) .
\end{aligned}
$$

The prime denotes conjugate patches. Note that, in contrast with Ref. [16], the equation for intrapatch order parameter is diagonal. This is because the Umklapp processes $g_{3}$ are forbidden. This excludes all symmetries of the superconductive order parameters, except from $s$ and $s^{ \pm}$. This is a major difference with respect to results on single-layer graphene. The intrapatch order parameter has $s$-wave symmetry and is given by

$$
\alpha_{1 s}=-2 d_{4} G_{4} .
$$

However, for the interpatch order parameter, we find both $s$ wave and $s^{ \pm}$-wave symmetry. The former is given by

$$
\alpha_{2 s}=-2 d_{3}\left(G_{1}+G_{2}\right),
$$

for nonconjugate patches, and

$$
\alpha_{3 s}=-2 d_{4}\left(\tilde{G}_{1}+\tilde{G}_{2}\right)
$$




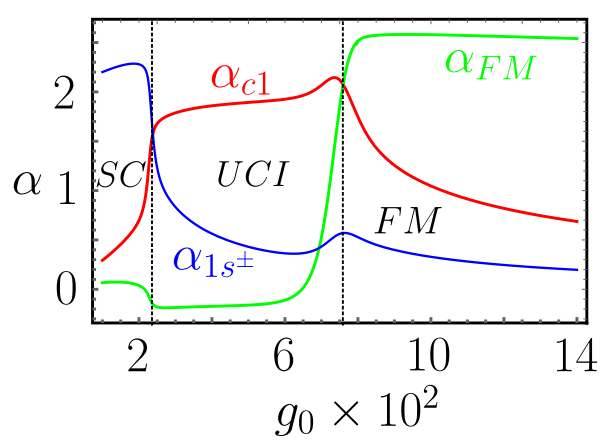

FIG. 6. Phase diagram of the system at Van Hove filling: at $g_{0}<$ $g_{0}^{(1)} \approx 0.024$, the leading instability is $s^{ \pm}$superconductivity (SC) with the order parameter changing sign along the path connecting two conjugate patches. At $g_{0}^{(1)}<g_{0}<g_{0}^{(2)} \approx 0.075$, the most rapidly divergent is uniform charge susceptibility leading to uniform charge instability phase (UCI), and at $g_{0}>g_{0}^{(2)}$ the leading instability is due to ferromagnetism (FM).

for conjugate patches. For the $s^{ \pm}$order parameter we find

$$
\alpha_{1 s^{ \pm}}=2 d_{4}\left(\tilde{G}_{1}-\tilde{G}_{2}\right)
$$

for conjugate patches, and

$$
\alpha_{2 s^{ \pm}}=2 d_{3}\left(G_{1}-G_{2}\right)
$$

for nonconjugate patches. In Figs. 5 and 6, we compare $\alpha_{i}$ for all potential instabilities at $\mu=0$ and for $g_{i}(0)=g_{0}>0$. The leading instability at $g_{0}<g_{0}^{(1)}$ is interpatch $s^{ \pm}$superconductivity corresponding to the coupling between conjugate patches with the order parameter changing signs along the path connecting two conjugate patches (see Fig. 6). For $g_{0}^{(1)}<$ $g_{0}<g_{0}^{(2)}$, the most divergent is the uniform charge susceptibility, which corresponds to the UCI phase, often referred to as phase separation (PS) between two states with different electronic densities as has been observed around transitions to a Mott insulating state [25-30] (see Fig. 6). And, finally, for $g_{0}>g_{0}^{(2)}$, the leading instability is due to ferromagnetism. The dependence of $\alpha_{i}$ on the initial condition $g_{0}$ might seam unusual; however, as we show in Appendix A, this can be explained by the dependence of the parameters $d_{i}$ on $y_{c}$ and consequently, on $g_{0}$.

For finite chemical potential, i.e., away from the Van Hove point, the RG Eqs. (16)-(22), as well the susceptibilities Eqs. (25)-(44) do not change; however, we use the approximation Eq. (23) for the parameter $d_{4}$. This allows us to compare the susceptibilities for different order parameters as a function of $z=\ln (\Lambda /|\mu|)$. In Fig. 7, we show $\alpha_{i}$ for $g_{0}=0.055$, corresponding to the UCI phase under the Van Hove doping. We found that for $z<z_{c} \approx 7.5$, the leading instability is the interpatch $s^{ \pm}$superconductivity corresponding to the coupling between conjugate patches with the order parameter changing sign along the path connecting two conjugate patches, followed by the UCI state at $z>z_{c}$. To estimate the transition temperature, we use the same approximation as for Eq. (23) and cast $y_{c}$ as $y_{c}=\frac{x_{c} z}{z-x_{c}}$, where $x_{c}=\ln \left(\Lambda / T_{c}\right)$. We then evaluate $y_{c}$ as a function of $z$ and extract $T_{c}$. The chemical potential can be calculated in terms of doping electronic
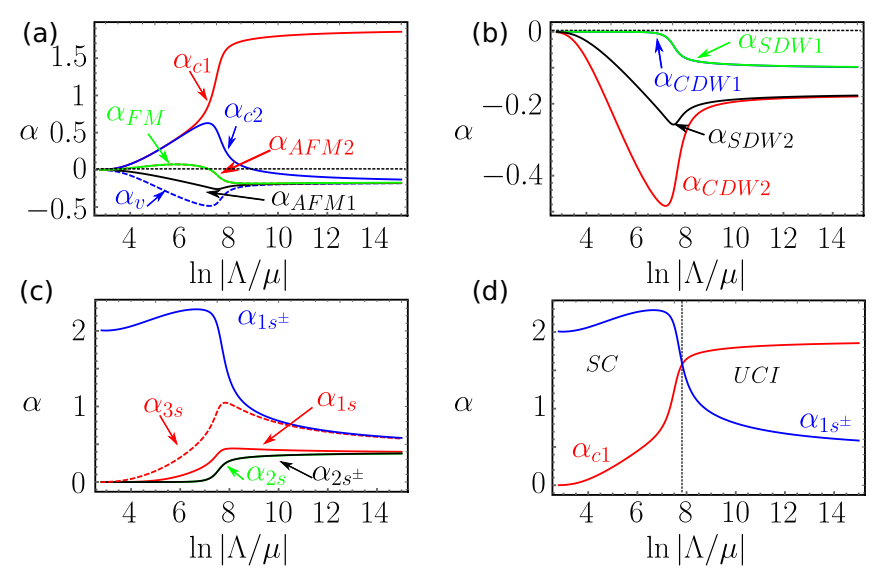

(d)

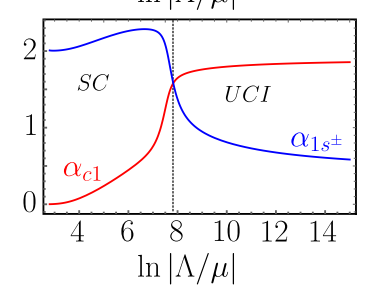

FIG. 7. Parameters of susceptibilities close to Van Hove filling for $g_{0}=0.055$. (a)-(c) $\alpha_{i}$ as functions of $z=\ln |\Lambda / \mu|$. (d) Leading instabilities as functions of $z$ showing the two dominating phases: $s^{ \pm}$superconductivity (SC) with the order parameter changing sign along the path connecting the two conjugate patches and the uniform charge instability phase (UCI) at $z_{c} \approx 7.5$.

density, $n$, as [1] $\mu=\hbar v^{*} \sqrt{n \pi / 2}-\epsilon_{0}$, for which the Van Hove doping is determined by the electronic density $n_{0}=$ $\frac{m^{2} v^{* 2}}{2 \pi \hbar^{2}}$. This allows us to present our result as the phase diagram in Fig. 8. Note that the symmetry of the phase diagram is due to the fact that $\mu$ changes sign at $n=n_{0}$; however, it is $|\mu|$ but not $\mu$ that enters all the expression for the polarization operators. Note that the width of the UCI phase is determined by the value of the coupling constant $g_{0}$, in accordance with Fig. 6. For $g_{0}<g_{0}^{(1)}$, the UCI phase is absent, and the system becomes superconducting for all doping charge densities close to $n_{0}$. At $g_{0}=g_{0}^{(1)}$, the UCI phase appears at $n=n_{0}$ and expands as $g_{0}$ grows. For $g_{0} \gtrsim g_{0}^{(2)}$, the ferromagnetic phase appears at $n \simeq n_{0}$.

\section{DISCUSSION}

The physics and the corresponding phase diagram in Fig. 8 is relevant to the experimental results of Ref. [2] obtained

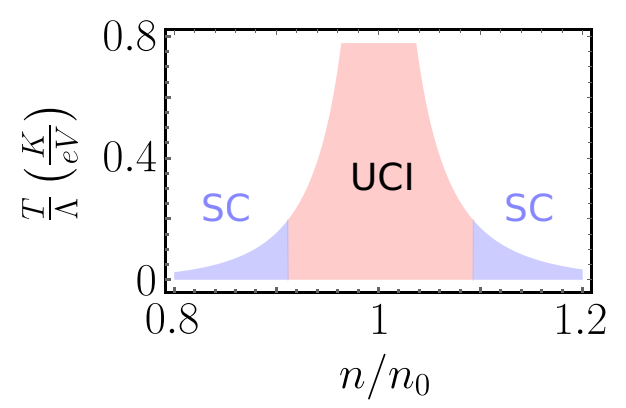

FIG. 8. Phase diagram for the system close to the Van Hove filling as a function of doping charge density, $n$, relative to the doping charge density, $n_{0}$, corresponding to the Van Hove filling for the set of parameters $g_{0}=0.055$ and $\epsilon_{0}=m v^{* 2} / 2=8 \times 10^{-3} \Lambda$. The two $s^{ \pm}$superconducting phases, with the order parameter changing sign along the path connecting two conjugate patches, are separated by a phase with a uniform charge instability. 
for $v=1$ per moiré cell. In the region near $n_{0}$, our results suggest that there is a phase of a UCI, identified as the state of PS. In the context of Hubbard model, this state of PS is seen in a number of reported calculations using a variety of techniques [25-30]. As a result of the coexistence of metallic and insulating regions, the transport in this particular phase is through percolation. Therefore, it is possible that in the range of values of densities and temperature it can display insulating behavior. This phase must be investigated further. The results have been verified by an alternative way of using the RG procedure as summarized in Appendix A.

In the case when the intervalley coupling, $g_{0}^{\text {inter }}$, is negligible in comparison with the intravalley one, $g_{0}^{\text {intra }}$, the system can be modeled by taking into account only the fermions living in three patches around Van Hove singularities that belong to the same valley. In this case, the two-fermion scattering is described by three distinct interactions in the low-energy theory, $g_{1}, g_{2}$, and $g_{4}$. The energy dispersions in the patches are all distinct and given by Eqs. (5). In this case, as we show in Appendix B, the phase diagram is similar to the one in Fig. 8: the UCI phase is sandwiched between the two superconducting phases (see Fig. 11 in Appendix B). However, the symmetry of the order parameter of the SC phase is now different. We predict that in the case $g_{0}^{\text {inter }} \ll g_{0}^{\text {intra }}$, it is of $s^{++}-$symmetry, in contrast with $s^{ \pm}$ order parameter predicted for $g_{0}^{\text {inter }}=g_{0}^{\text {intra }}$, i.e., it does not change sign along the path connecting two patches. Note also that in our calculations we considered only nearest-neighbor interactions corresponding to particle-hole symmetry. Taking into account further neighbors in interaction will break the particle hole symmetry; however, it will not change the results qualitatively.

In conclusion, we have investigated a microscopic model that takes into account interactions among electrons that live around the points of Van Hove singularities formed in twisted bilayer graphene near filling factor $v=1$ and found the different phases as a function of electron density. We found superconductivity which displays a reentrant behavior as a function of electronic density. We predict that the order parameter symmetry is $s^{ \pm}$, while, if the intervalley coupling is negligible, it is of $s^{++}$symmetry. The phase in the middle of the two superconducting phases is characterized by a divergence of charge susceptibility at $q=0$. This signals a phase of coexistence of metallic and insulating regions in a PS. The other phase that appears in the phase diagram is the ferromagnetic one. It is worth mentioning that the width of the two superconducting phases can be tuned according to the values of $g_{0}$, therefore extending the regions. As a result, the physics associated with different filling factors is quite different, as different singularities in DOS and different nesting conditions exist. Our theory can accommodate recent experiments [31] where pressure has been used to tune the system, which is work in progress.

\section{ACKNOWLEDGMENTS}

We are grateful to Andrey Chubukov for his questions and comments that led us to verify the results using an alternative RG procedure. We also thank Dima Efremov, Mark Greenaway, and Andreas Rost for useful discussions on related
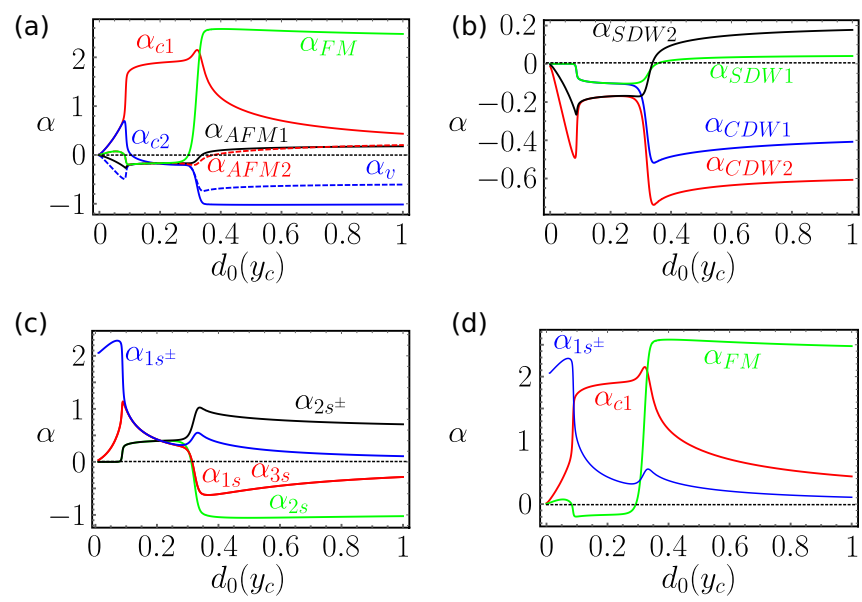

FIG. 9. (a)-(c) Parameters of susceptibilities at the Van Hove filling as functions of $d_{0}\left(y_{c}\right)$ for $g_{i}(0)=g_{0}>0$ and equal inter- and intravalley scatterings $g_{0}^{\text {inter }}=g_{0}^{\text {intra }}$. (d) Phase diagram with leading instabilities corresponding to $s^{ \pm}$-wave superconductivity, UCI phase, and FM phase.

problems. This work was supported by the EPSRC through Grant No. EP/P002811/1.

\section{APPENDIX A: ALTERNATIVE RG EQUATIONS}

Alternatively, we can obtain the RG equations by differentiating $g_{i}$ with respect to the RG "time", $y=2 \Pi_{p p}(0) / \nu_{0}$, which, for $|\mu| \ll T$, is quadratic in $\ln |\Lambda / T|$. This procedure leads again to the same equations Eqs. (16)-(22) with different parameters $d_{i}: d_{0}=y^{-1 / 2}, d_{1}=A y^{-1 / 2}, d_{3}=B y^{-1 / 2}$, and $d_{4}=1$. We solve Eqs. (16)-(22) with the new parameters $d_{i}$, following the method of Ref. [16]; because the RG equations flow to strong coupling at a finite scale $y_{c}$, we treat $d_{0}\left(y_{c}\right)$ as a parameter in our calculations. However, in contrast with Ref. [16], we keep the subleading terms, $d_{1}$ and $d_{3}$, and assume that $d_{1} / d_{0}=A=$ const and $d_{3} / d_{0}=B=$ const. We then calculate $g_{i}=\frac{G_{i}}{y_{c}-y}$ and find the susceptibilities as a function of $d_{0}\left(y_{c}\right)$. The results are shown in Fig. 9. The phase diagram that is extracted from the results of this method Fig. 9(d), has the same structure as the one presented in Fig. 6: a UCI phase is sandwiched between the $s^{ \pm}$superconducting phase and the FM-phase. We also find that $y_{c} \propto g_{0}^{-1}$, which explains the dependence of $\alpha_{i}$ on $g_{0}$ in Fig. 6: the solutions of Eqs. (16)-(22) with constant $d_{i}$ are independent of $g_{0}$, however, $d_{0}$ does depend on $g_{0}$ as $d_{0} \propto \sqrt{g_{0}}$, which, in turn, carries the dependence on the initial condition to $g_{i}$ and $\alpha_{i}$.

\section{APPENDIX B: THE CASE OF NEGLIGIBLE INTERVALLEY SCATTERING}

Here we consider the limit of the screened Coulomb interaction, $U(\mathbf{k})$, being short-ranged in the $k$-space, i.e., $U\left(\Delta \mathbf{K}_{s}\right) \ll U(0)$. In this limit, the intervalley scattering is negligible, and the low-energy Lagrangian can be expressed in terms of the three distinct interactions, $g_{1}, g_{2}$, and $g_{4}$, coupling 
(a)
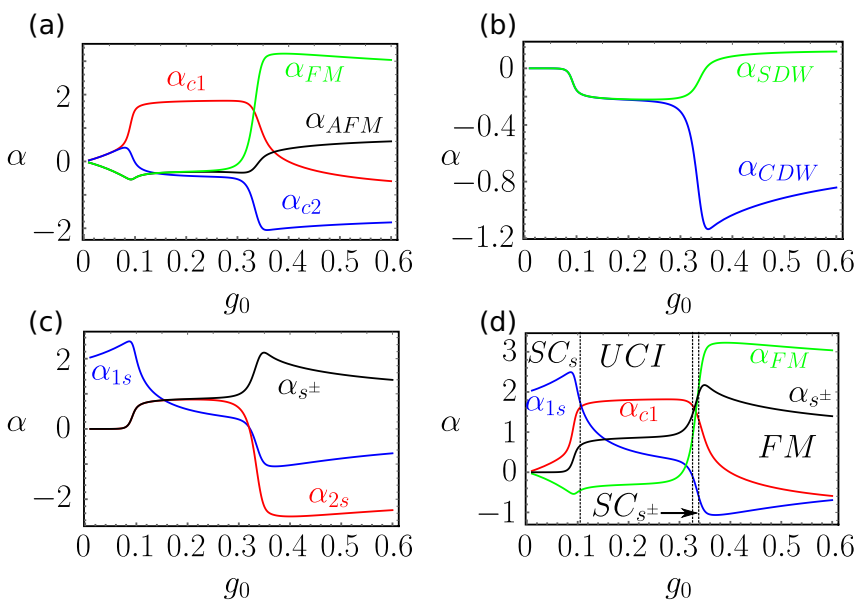

FIG. 10. (a)-(c) Parameters of susceptibilities at Van Hove filling as functions of $g_{i}(0)=g_{0}>0$ for $g_{0}^{\text {inter }} \ll g_{0}^{\text {intra }}$. (d) Phase diagram with leading instabilities corresponding to $s$-wave superconductivity $\left(\mathrm{SC}_{s}\right), \mathrm{UCI}$ phase, $s^{ \pm}$-superconductivity $\left(\mathrm{SC}_{s^{ \pm}}\right)$, and $\mathrm{FM}$ phase.

only the patches belonging to the same valley:

$$
\begin{aligned}
\mathcal{L}= & \sum_{\alpha, \sigma} \psi_{\alpha \sigma}^{\dagger}\left(\partial_{\tau}-\epsilon_{k}+\mu\right) \psi_{\alpha \sigma}-\frac{1}{2} \sum_{\alpha, \beta, \sigma, \sigma^{\prime}}\left[g_{1} \psi_{\alpha \sigma}^{\dagger} \psi_{\beta \sigma^{\prime}}^{\dagger}\right. \\
& \left.\times \psi_{\alpha \sigma^{\prime}} \psi_{\beta \sigma}+g_{2} \psi_{\beta \sigma}^{\dagger} \psi_{\alpha \sigma^{\prime}}^{\dagger} \psi_{\alpha \sigma^{\prime}} \psi_{\beta \sigma}\right] \\
& -\frac{1}{2} \sum_{\alpha, \sigma, \sigma^{\prime}} g_{4} \psi_{\alpha \sigma}^{\dagger} \psi_{\alpha \sigma^{\prime}}^{\dagger} \psi_{\alpha \sigma^{\prime}} \psi_{\alpha \sigma}
\end{aligned}
$$

where patches $\alpha$ and $\beta$ belong to the same valley.

We compare the susceptibilities for the case of $\mu=0$ in Fig. 10. We found that for $g_{0} \lesssim 0.1$, the leading instability is intra-patch $s$-wave superconductivity, followed by UCI phase for $0.1 \lesssim g_{0} \lesssim 0.32, s^{ \pm}$-wave superconductivity at the narrow range $0.32 \lesssim g_{0} \lesssim 0.34$, and, finally, FM phase for $g_{0} \gtrsim 0.34$, as we show in Fig. 10(d).

Using the Feynman diagrams in Figs. 3(a) and 3(b), we obtain the following RG equations in the one-loop approximation:

$$
\begin{aligned}
& \frac{d g_{1}}{d y}=g_{1}\left(g_{1}+2 g_{4}\right)+2 d_{1} g_{1}\left(g_{2}-g_{1}\right)-2 d_{3} g_{1} g_{2} \\
& \frac{d g_{2}}{d y}=2\left(g_{1} g_{4}+g_{1} g_{2}-g_{2} g_{4}-g_{2}^{2}\right)+d_{1} g_{2}^{2}-d_{3}\left(g_{1}^{2}+g_{2}^{2}\right) \\
& \frac{d g_{4}}{d y}=g_{4}^{2}+2 g_{1}^{2}-4 g_{2}^{2}+4 g_{1} g_{2}-d_{4}(y) g_{4}^{2} .
\end{aligned}
$$

Next, we introduce the test vertices in the same way as in the main text, and derive their renormalization using Feynman

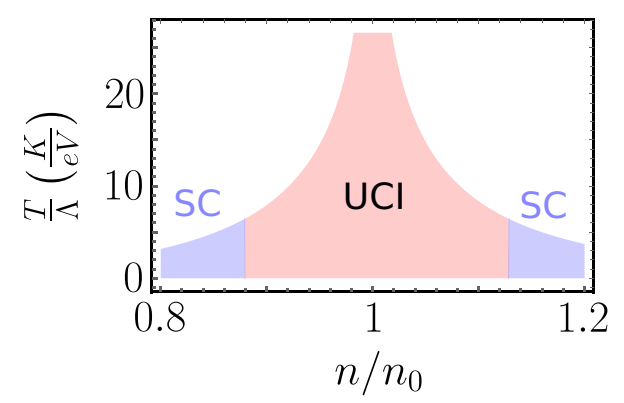

FIG. 11. Phase diagram for the system with $g_{0}^{\text {inter }} \ll g_{0}^{\text {intra }}$ close to the Van Hove filling as a function of doping charge density, $n$, relative to the doping charge density, $n_{0}$, corresponding to the Van Hove filling for the set of parameters $g_{0}=0.2$ and $\epsilon_{0}=m v^{* 2} / 2=10^{-2} \Lambda$. The two intrapatch $s$-wave superconducting phases are separated by a phase with a uniform charge instability.

diagrams in Fig. 3(c). For the instabilities due to uniform densities, we find

$$
\frac{d n_{\sigma \alpha}}{d y}=-g_{4} n_{\bar{\sigma} \alpha}+\sum_{\beta \neq \alpha}\left[\left(g_{1}-g_{2}\right) n_{\sigma \beta}-g_{2} n_{\bar{\sigma} \beta}\right],
$$

where $\beta$ and $\alpha$ denote the patches belonging to the same valley. We find four distinct eigenvalues corresponding to charge, $\alpha_{c 1}$ and $\alpha_{c 2}$, antiferromagnetic, $\alpha_{\mathrm{AFM}}$, and ferromagnetic, $\alpha_{\mathrm{FM}}$, instabilities:

$$
\begin{gathered}
\alpha_{c 1}=2\left(2 G_{1}-4 G_{2}-G_{4}\right), \\
\alpha_{c 2}=2\left(-G_{1}+2 G_{2}-G_{4}\right), \\
\alpha_{\mathrm{AFM}}=2\left(-G_{1}+G_{4}\right), \\
\alpha_{\mathrm{FM}}=2\left(2 G_{1}+G_{4}\right) .
\end{gathered}
$$

For the CDW and SDW instability, the vertex renormalization is given by Eq. (31), with the CDW and SDW susceptibilities, $\alpha_{\mathrm{CDW}}$, and, $\alpha_{\mathrm{SDW}}$, given by Eqs. (33) and (35), respectively. Finally, the renormalization of the superconducting test vertices are given by Eqs. (37) and (38) for intraand interpatch superconductivity respectively, leading to the two susceptibilities with $s$-wave order parameter, $\alpha_{1 s}$ and $\alpha_{2 s}$, given by Eqs. (40) and (41), respectively, as well as the susceptibility with $s^{ \pm}$order parameter, $\alpha_{s^{ \pm}}$, given by Eq. (44).

The phase diagram for the system with finite $\mu$ as a function of doping charge density shown in Fig. 11 is similar to the one obtained for the case of valley-independent scattering. However, in the case of negligible intervalley scattering, the superconducting phase has $s^{++}$order parameter in contrast with the case of valley-independent scattering, where the order parameter is $s^{ \pm}$.
[1] Y. Cao, V. Fatemi, A. Demir, S. Fang, S. L. Tomarken, J. Y. Luo, J. D. Sanchez-Yamagishi, K. Watanabe, T. Taniguchi, E. Kaxiras, R. C. Ashoori, and P. Jarillo-Herrero, Correlated insulator behavior at half-filling in magic-angle graphene superlattices, Nature 556, 80 (2018).
[2] Y. Cao, V. Fatemi, S. Fang, K. Watanabe, T. Taniguchi, E. Kaxiras, and P. Jarillo-Herrero, Unconventional superconductivity in magic-angle graphene superlattices, Nature 556, 43 (2018).

[3] G. Li, A. Luican, J. M. B. Lopes dos Santos, A. H. Castro Neto, A. Reina, J. Kong, and E. Y. Andrei, Observation of Van 
Hove singularities in twisted graphene layers, Nat. Phys. 6, 109 (2010).

[4] Y. Sherkunov, A. V. Chubukov, and J. J. Betouras, Effects of Lifshitz Transitions in Ferromagnetic Superconductors: The Case of URhGe, Phys. Rev. Lett. 121, 097001 (2018).

[5] C. Liu, T. Kondo, R. M. Fernandes, An D. Palczewski, E. D. Mun, N. Ni, A. N. Thaler, A. Bostwick, E. Rotenberg, J. Schmalian, S. L. Bud'ko, P. C. Canfield, and A. Kaminski, Evidence for a Lifshitz transition in electron-doped iron arsenic superconductors at the onset of superconductivity, Nat. Phys. 6, 419 (2010).

[6] S. Slizovskiy, A. V. Chubukov, and J. J. Betouras, Magnetic Fluctuations and Specific Heat in $\mathrm{Na}_{x} \mathrm{CoO}_{2}$ Near a Lifshitz Transition, Phys. Rev. Lett. 114, 066403 (2015).

[7] B. Padhi, C. Setty, and P. W. Phillips, Doped twisted bilayer graphene near magic angles: Proximity to Wigner crystallization, not Mott insulation, Nano Lett. 18, 6175 (2018).

[8] P.A. Lee, N. Nagaosa, and X.G. Wen, Doping a Mott insulator: Physics of high-temperature superconductivity, Rev. Mod. Phys. 78, 17 (2006).

[9] C. Xu and L. Balents, Topological Superconductivity in Twisted Multilayer Graphene, Phys. Rev. Lett. 121, 087001 (2018).

[10] H. C. Po, L. Zou, A. Vishwanath, and T. Senthil, Origin of Mott Insulating Behavior and Superconductivity in Twisted Bilayer Graphene, Phys. Rev. X 8, 031089 (2018).

[11] J. F. Dodaro, S. A. Kivelson, Y. Schattner, X. Q. Sun, and C. Wang, Phases of a phenomenological model of twisted bilayer graphene, Phys. Rev. B 98, 075154 (2018).

[12] H. Isobe, N. F. Q. Yuan, and L Fu, Unconventional superconductivity and density waves in twisted bilayer graphene, arXiv: 1805.06449 .

[13] C.-C. Liu, L.-D. Zhang, W.-Q. Chen, and F. Yang, Chiral SDW and $\mathrm{d}+\mathrm{id}$ superconductivity in the magic-angle twisted bilayergraphene, arXiv:1804.10009.

[14] Y.-Z. You and A. Vishwanath, Superconductivity from valley fluctuations and approximate $\mathrm{SO}(4)$ symmetry in a weak coupling theory of twisted bilayer graphene, arXiv:1805.06867.

[15] B. Uchoa and A. H. Castro Neto, Superconducting States of Pure and Doped Graphene, Phys. Rev. Lett. 98, 146801 (2007).

[16] R. Nandkishore, L. S Levitov, and A. V. Chubukov, Chiral superconductivity from repulsive interactions in doped graphene, Nat. Phys. 8, 158 (2012).
[17] J. M. B. Lopes dos Santos, N. M. R. Peres, and A. H. Castro Neto, Graphene Bilayer with a Twist: Electronic Structure, Phys. Rev. Lett. 99, 256802 (2007).

[18] E. J. Mele, Commensuration and interlayer coherence in twisted bilayer graphene, Phys. Rev. B 81, 161405(R) (2010).

[19] E. J. Mele, Band symmetries and singularities in twisted multilayer graphene, Phys. Rev. B 84, 235439 (2011).

[20] R. Bistritzer and A. H. MacDonald, Moiré bands in twisted double-layer graphene, Proc. National Acad. Sci. 108, 12233 (2011).

[21] A. Shtyk, G. Goldstein, and C. Chamon, Electrons at the monkey saddle: A multicritical Lifshitz point, Phys. Rev. B 95, 035137 (2017).

[22] N. Furukawa, T. M. Rice, and M. Salmhofer, Truncation of a Two-Dimensional fermi Surface Due to Quasiparticle Gap Formation at the Saddle Points, Phys. Rev. Lett. 81, 3195 (1998).

[23] K. Le Hur and T. M. Rice, Superconductivity close to the Mott state: From condensed-matter systems to superfluidity in optical lattices, Ann. Phys. 324, 1452 (2009).

[24] J. González, Kohn-Luttinger superconductivity in graphene, Phys. Rev. B 78, 205431 (2008).

[25] G. Kotliar, S. Murthy, and M. J. Rozenberg, Compressibility Divergence and the Finite Temperature mott Transition, Phys. Rev. Lett. 89, 046401 (2002).

[26] M. Aichhorn, E. Arrigoni, M. Potthoff, and W. Hanke, Phase separation and competition of superconductivity and magnetism in the two-dimensional Hubbard model: From strong to weak coupling, Phys. Rev. B 76, 224509 (2007).

[27] A. Macridin, M. Jarrell, and Th. Maier, Phase separation in the Hubbard model using the dynamical cluster approximation, Phys. Rev. B 74, 085104 (2006).

[28] M. Eckstein, M. Kollar, M. Potthoff, and D. Vollhardt, Phase separation in the particle-hole asymmetric Hubbard model, Phys. Rev. B 75, 125103 (2007).

[29] T. Misawa and M. Imada, Origin of high- $T_{c}$ superconductivity in doped Hubbard models and their extensions: Roles of uniform charge fluctuations, Phys. Rev. B 90, 115137 (2014).

[30] J. Otsuki, H. Hafermann, and A. I. Lichtenstein, Superconductivity, antiferromagnetism, and phase separation in the twodimensional Hubbard model: A dual-fermion approach, Phys. Rev. B 90, 235132 (2014).

[31] M. Yankowitz, S. Chen, H. Polshyn, K. Watanabe, T. Taniguchi, D. Graf, A.F. Young, and C. R. Dean, Tuning superconductivity in twisted bilayer graphene, arXiv:1808.07865. 